\title{
Interleukin-6 in Patients with Cardiac Myxoma
}

\author{
Shi-Min Yuan ${ }^{1}$ and Hui-Zhen Lin ${ }^{2}$ \\ ${ }^{1}$ Department of Cardiothoracic Surgery, The First Hospital of Putian, Teaching Hospital, Fujian Medical University, Putian, Fujian \\ Province, China \\ ${ }^{2}$ Department of Clinical Laboratory, The First Hospital of Putian, Teaching Hospital, Fujian Medical University, Putian, Fujian Province, \\ China
}

\begin{abstract}
The relationships between interleukin (IL)- 6 and cardiac myxoma remain to be clarified. This article systematically reviewed the IL-6 properties in cardiac myxoma patients based on retrieval of pertinent literature published between 1998 and 2018. Significant differences were found in circulating IL- 6 values between preoperation and 1 and 6 months after operation. Preoperative circulating IL-6 correlated significantly with tumour volume $(r=0.8552, p=0.003)$, while there were no significant correlations with maximal tumour dimension $(r=0.2443, p=0.190)$. No correlation was found between circulating IL- 6 at 1 and 6 months after tumour resection with either tumour volume or with maximal tumour dimension. The positive rate of immunostaining of IL-6 in cardiac myxoma tissues was 93.3\%. Overproduction of IL-6 is responsible for the inflammatory presentations, constitutional symptoms, and recurrence and distal embolisation of cardiac myxoma. Cardiac myxoma could be a cellular source of IL- 6 release. Cardiac myxoma resection is an absolute choice of eliminating IL-6 production in these patients.
\end{abstract}

Key Words: Cardiac surgical procedures, Cytokines; Inflammation, Interleukin-6, Neoplasms.

How to cite this article: Yuan SM, Lin HZ. Interleukin-6 in Patients with Cardiac Myxoma. J Coll Physicians Surg Pak 2020; 30(08):849-852.

\section{INTRODUCTION}

The interleukin (IL) family is a group of cytokines involved in the pathogenesis of various inflammation-related diseases, allergic disorders, infections, immune deficiencies, tumours, tissue fibrosis, and hypoxic conditions. Of the cardiac surgical patients, those with a cardiac myxoma showed particular inherentrelationships to IL-6. ${ }^{1,2}$

It has been recognised that IL- 6 is responsible for the constitutional presentations, ${ }^{3}$ tumour recurrence, ${ }^{4}$ and distal embolisation of patients with cardiac myxoma. ${ }^{5}$ Surgical resection of cardiac myxoma may lead to a quick reduction of circulating IL- $6 .{ }^{5}$ Even though sporadic reports have been constantly published on the relations between IL- 6 and cardiac myxoma, an overall statement of this respect is still scanty.

The aim of this research was to present the gathered evidence regarding IL-6 profiles in cardiac myxoma patients.

Correspondence to: Shi-Min Yuan, Department of Cardiothoracic Surgery, The First Hospital of Putian, Teaching Hospital, Fujian Medical University, 389 Longdejing Street, Chengxiang District, Putian 351100, Fujian

Province, People's Republic of China

E-mail: shiminyuan@126.com

Received: March 13, 2019; Revised: August 07, 2019;

Accepted: September 25, 2019

DOI: https://doi.org/10.29271/jcpsp.2020.08.849

\section{METHODOLOGY}

The preferred reporting items for systematic reviews and meta-analyses (PRISMA) statement guidelines were followed in this meta-analysis. Publications were systematically searched in the PubMed, Highwire Press, and the Cochrane Library databases between 1998 and 2018. The MeSH terms and keywords used to identify articles included "interleukin-6", "cardiac myxoma", "atrial myxoma", "ventricular myxoma", "valvular myxoma", "plamsa/serum", and "immunohistochemistry". Bibliographic references were also tracked for the completeness of the literature retrieval. Thirty-eight articles were found related to the topic and keywords in the literature search; and 13 articles, which met the inclusion criteria during preliminary assessment, were included in the review. The exclusion criteria were: unrelative to IL-6 and cardiac myxoma $(n=9)$, other biomarkers of patients with cardiac myxoma $(n=6)$, general discussions of IL- 6 and cardiac myxoma $(n=5)$, and IL- 6 in patients with a cardiac tumour other than cardiac myxoma $(n=5)$.

Data were carefully extracted for details of the study population, demographics, constitutional symptoms, comorbidities, locations and dimensions of cardiac myxoma, serum/plasma IL-6 values, positivity of IL- 6 immunostaining, treatment, and patient's outcomes, etc.

Quantitative data were presented as mean \pm standard deviation. The intergroup differences were compared by independent samples t-test, and comparisons of frequencies were made by Pearson's Chi-square test. Correlation was assessed 
between IL-6 levels and tumour size. $p<0.05$ was considered statistically significant. IBM SPSS statistics 22.0 was used for conducting the statistical analyses.

\section{RESULTS}

Comprehensive literature retrieval resulted in 13 pertinent articles describing IL-6 in cardiac surgical patients. ${ }^{4-16}$ There were 53 patients with 35 (66.0\%) female and $18(34.0 \%)$ male patients. A female predominance was found $\left(\chi^{2}=10.9\right.$, $p=0.001$ ) with a female-to-male ratio of $1.9: 1$. Their mean age was $48.3 \pm 20.3$ years $(n=34)$.

Twelve (22.6\%) patients had constitutional symptoms. , $^{4,7-9,13,14,16}$ A sequal of cardiac myxoma was found in $8(15.1 \%)$ patients: cerebral infarct $(n=4), 8,9,12$ cerebral aneurysm $(n=2),{ }^{5,6}$ cerebral embolism $(n=1),{ }^{12}$ and systemicembolism $(n=1){ }^{10}$

This patient setting had 59 myxomas: 49 (83.1\%) were in the left atrium, ${ }^{4-13,15,16}$ four $(6.8 \%)$ in the right atrium, ${ }^{12,15}$ three (5.1\%) from the mitral valve, ${ }^{4,15}$ two $(3.4 \%)$ in the right ventricle, ${ }^{15}$ and one (1.7\%) in the left ventricle. ${ }^{15}$ Yokomuro et al. reported 25 cardiac myxomas in 21 patients without indicating exactly the situations of solitory and multiple cardiac myoxmas. ${ }^{15}$ Thus, it was unable to make a comparative analysis between patients with a solitary and those with multiple cardiac myxomas in the presentstudy.

The maximal dimension of cardiac myxoma of the patient population was $49.3 \pm 19.9 \mathrm{~mm}(\mathrm{n}=44)$. The dimension of the left cardiac myxoma was $52.5 \pm 18.8 \mathrm{~mm}(n=29)$. The volume of the cardiac myxoma was 106,976.4 $\pm 196,027.2 \mathrm{~mm}^{3}(\mathrm{n}=23)$.

The detection method of circulating IL- 6 was described in only 2 (15.4\%) of the 13 reports. ${ }^{4,9}$ Enzyme-linked immunosorbent assay (ELISA) was used in both reports.

Preoperative circulating IL-6 was detected in 30 patients: it was elevated in $26(86.7 \%)$ patients and normal in 4 (13.3\%) patient $\left(\chi^{2}=32.3, p<0.001\right)$. The preoperative IL-6 value was $26.1 \pm 28.5$ $\mathrm{pg} / \mathrm{mL}(\mathrm{n}=17)$. Postoperative circulating IL- 6 at 0.5 month was $5.3 \mathrm{pg} / \mathrm{mL}(\mathrm{n}=1) .{ }^{13}$ At 1 month, 13 patients had their circulating IL-6 detected; it was normal in 12 (92.3\%) patients and it was still high in 1 (7.7\%) patient. ${ }^{6}$ The mean value of IL- 6 was $5.8 \pm 7.7$ $\mathrm{pg} / \mathrm{mL}(n=13)$.

All 8 patients whose circulating IL- 6 was detected at 6 months had a normal IL- 6 value of $2.6 \pm 1.1 \mathrm{pg} / \mathrm{mL}(n=7)$. The circulating IL- 6 values at 1 month and 6 months after operation were significantly lower than preoperation ( $p=0.019$ and $p=0.043$, respectively). No significant difference was found in the circulating IL- 6 value between 1 month and 6 months after the operation $(p=0.290)$. Two patients with a recurrent cardiac myxoma had extremely high circulating IL-6 levels, up to 77 and $102 \mathrm{pg} / \mathrm{mL}$, respectively. ${ }^{4}$ Preoperative C-reactive protein was $8.2 \pm 19.8$ $\mathrm{mg} / \mathrm{dL}(\mathrm{n}=23)$. No significant correlation was found between preoperative IL-6 and C-reactive protein $(r=0.2958, p=0.175)$.

No significant differences were found in IL-6 levels of preoperation, and 1 and 6 months after cardiac myxoma resection between patients with and without constitutional symptoms (preoperation: $36.0 \pm 32.5 \mathrm{pg} / \mathrm{mL}$ vs. $12.0 \pm 13.9 \mathrm{pg} / \mathrm{mL}$, $\mathrm{p}=0.087 ; 1$ month: $4.1 \pm 2.3 \mathrm{pg} / \mathrm{mL}$ vs. $7.8 \pm 12.6 \mathrm{pg} / \mathrm{mL}$, $\mathrm{p}=0.466$; and 6 months: $2.5 \pm 0.9 \mathrm{pg} / \mathrm{mL}$ vs. $2.8 \pm 1.5 \mathrm{pg} / \mathrm{mL}$, $p=0.783)$. Even though, there was a disparity in the preoperative values between the two groups. Patients with cerebral comobidities had higher IL- 6 levels before operation and 1 month after cardiac myxoma resection than patients without (preoperation: $36.7 \pm 49.2 \mathrm{pg} / \mathrm{mL}$ vs. $21.7 \pm 15.3 \mathrm{pg} / \mathrm{mL}$, $\mathrm{p}=0.340$; and 1 month: $15.0 \pm 21.2 \mathrm{pg} / \mathrm{mL}$ vs. $3.8 \pm 2.1 \mathrm{pg} / \mathrm{mL}$, $p=0.066)$, but lack of a statistical significance.

Preoperative circulating IL-6 correlated significantly with tumour volume $(r=0.8552, p=0.003)$, while there were no significant correlations with maximal tumour dimension $(r=0.2443, p=0.190)$. Circulating IL-6 at 1 month after tumour resection showed no correlation with either tumour volume $(r=0.5132, p=0.097)$ or with maximal tumour dimension $(r=-0.1729, p=0.2955)$.

Neither a correlation was found between circulating IL-6 at 6 month after tumour resection with either tumour volume ( $r=-0.2992, p=0.257$ ) or with maximal tumour dimension $(r=-0.2759, p=0.275)$.

An immunohistochemical study was performed in 30 (56.6\%) patients. A positive reaction was observed in $28(93.3 \%)$ and negative in $2(6.7 \%)$ patients. In $16(57.1 \%)$ patients with a positive immunohistochemical reaction, the intensities of the staining were expressed semiqualitatively as + in $4(25 \%)$, $9,14,16$ $1+$ in $3(18.8 \%),{ }^{12} 2+$ in $2(12.5 \%),{ }^{12} 3+$ in $6(37.5 \%)$ patients, ${ }^{12,15}$ and $4+\operatorname{in} 1$ (6.3\%) patient. ${ }^{12}$ In 12 (42.9\%) of the 28 patients with a positive reaction to IL-6, the immunohistochemical intensities were expressed quatitatively, as $17.8 \pm 19.8 \%(n=13) .{ }^{15}$ Only one $(1.9 \%)$ patient was reported to be died, but the patient's detailed information was unavailable. ${ }^{4}$

\section{DISCUSSION}

Interleukin-6 is a multifactorial cytokine that causes differentiation and proliferation of normal and malignant cells, induction of the acute phase response and fever. Myxoma is the only cardiac tumour that causes increased circulating IL-6 levels, and no patient with other benign or malignant cardiac tumour was observed with an elevated IL- $6 .{ }^{17}$

IL-6 is known to increase serum concentrations of acute phase proteins and $\gamma$-globulin, favours the production of autoantibodies, and causes fever and even fever of unknown origin. ${ }^{17}$ It can be a possible explanation for the inflammatory and immune features in cardiac myxoma patients presenting with constitutional symptoms. Severe inflammatory response showed an elevation of preoperative serum IL-6 level up to $31.0 \mathrm{pg} / \mathrm{mL}^{13}$ Increases in serum IL-6, IL-6 antigen, and IL-6 mRNA expression in myxoma tissue were also reported in association with immunologic abnormalities. ${ }^{17}$ The positive rate of IL- 6 in cardiac myxoma patients as a whole was reported to be $46 \% .{ }^{17}$ Yokomuro et al. investigated the immunologic and serum IL-6 levels 
in 13 preoperative patients and in 10 postoperative patients. Preoperatively, $53.8 \%$ of the patients were positive for IL-6, and these values were found to normalise at 4 weeks after surgery. ${ }^{15}$ Endo et al. reported that, in cardiac myxoma patients presenting with constitutional symptoms, the positive rate of IL-6 was $92 \%$ in contrast to $0 \%$ of cardiac myxoma patients without any constitutional symptoms. ${ }^{17}$ As reported in a recent retrospective study, the preoperative IL- 6 levels did not have a difference between cardiac myxoma patients with and without constitutional symptoms, and the two group patients had very close IL- 6 levels. ${ }^{18}$ Whereas, there was a big gap of IL- 6 between patients with and without constitutional symptoms in this paper. The heterogenous results collected from the literature might explain the disparity of the two studies.

The paraneoplastic effects of cardiac myxoma may be related to tumour-secreted cytokines, including interleukins, implying tumour growth, recurrence, and distant metastasis. ${ }^{1}$ Mendoza et al. studied the correlation of IL-6 serum levels with preoperative constitutional symptoms and immunologic abnormalities, and the possible role played by this cytokine in tumour recurrence. ${ }^{4}$ They measured IL-6 serum levels by enzyme-linked immunosorbent assay method preoperatively, and 1 and 6 months after surgery in eight consecutive patients with nonfamilial myxoma. Two patients had a recurrent tumour, and one of them died during the second surgical operation for tumour recurrence. Their study also proved that patients with a first occurrence of cardiac myxoma showed a positive correlation between circulating IL- 6 values and tumour dimensions, but patients with recurrenttumourshad much higher IL-6 levelsirrespective of tumour size. Nevertheless, Yokomuro et al. reported that their patient with recurrent cardiac myxoma did not show elevated IL-6 values. ${ }^{15}$

It was also reported that overproduction of IL-6 was also observed in cardiac myxoma patients with cerebral aneurysm formation with a significant correlation. ${ }^{6}$ Serum IL-6 level extremely increased in patients with cardiac myxoma-induced cerebral aneurysms before surgery, normalised shortly after surgical resection of cardiac myxoma, but cerebral aneurysmal regression was not observed. ${ }^{5}$ A statistic analysis based on limited patient information revealed a much higher preoperative IL- 6 value than postoperative value in such patients, but lack of a significant difference $(30.1 \pm 25.9 \mathrm{pg} / \mathrm{mL}$ vs. $12.4 \pm 12.6$ $\mathrm{pg} / \mathrm{mL}, \mathrm{p}=0.266) .{ }^{19}$ Yaguchi et al. found that IL-6 levels in cerebrospinal fluid and in serum were concurrently elevated in a patient with cerebral aneurysm associated with cardiac myxoma. ${ }^{20}$ Mochizuki et al. observed that IL- 6 decreased 1 week after operation and was undetectable since 3 weeks. ${ }^{14}$ The immunohistochemical studies showed that the myxoma cells of their patients were positive to IL-6. Koo et al. reported a myxoma patient and a normal IL- 6 at 1 month after operation. ${ }^{5}$ Ezerioha and Feng reported a patient with cardiac myxoma and cerebral aneurysm, in whom IL-6 levels were significantly elevated before operation but persistently high at one month after operation. ${ }^{6}$ They concluded that some subsequent cerebral aneurysm was the cause of constant high IL-6.

Mendoza et al. demonstrated a positive correlation between serum IL-6 level and tumour size, and that overproduction of IL- 6 by myxoma cells were associated with tumour recurrence or distal embolisation. ${ }^{4}$ In the present study, a similar finding was illustrated in that preoperative IL-6 levels directly correlated with the tumour volume, but did not correlate with the maximaltumour dimension.

Acebo et al. illustrated the immunoreactivity to IL- 6 observed in 35 patients of cardiac myxoma. ${ }^{21}$ Granular cytoplasmic positivity was observed in 26 (74\%) myxomas. In cardiac myxoma patients with a positive reaction of myxoma cells with IL-6, a positive reaction was also observed in the lepidic, endothelial, smooth muscle cells and cardiomyocytes. Most patients with an abnormal laboratory parameters, such as anaemia, hypergammaglobulinemia, and increased erythrocyte sedimentation rate, had myxomas that expressed IL-6, although statistically this relationship was not significant. They found $76 \%$ of cardiac myxoma patients were positive for IL-6, and most of them had abnormal laboratory parameters. The irregular surface tumours are related to embolism, and large left atrial tumours are related to atrial fibrillation. Volz et al. found the immunohistochemical staining with anti-IL-6-antibody detected myxoma cells and scattered macrophages within the myxoma as the source of the elevated serum IL-6 levels. ${ }^{16}$

Moreover, intracellular expression of IL- 6 was found in $74.4 \%$ of monocytes in the peripheral blood before surgery; whereas, only $9 \%$ of monocytes were positive for IL- 6 at one month after tumour resection of cardiac myxoma. In contrast, the tumour necrosis factor- $\beta$ production was low and similar before and after surgery, which were $11.02 \%$ and $7.78 \%$, respectively. ${ }^{22}$

The imcomplete data source from the collected reports may have led to statistical bias in the present study. For example, comparisons of IL-6 expressions could not be made between left atrial myxomas and myxomas arising from other cardiac chambers, between patients with and without constitutional symptoms; and between solitary and multiple cardiac myxomas. Prospective studies with large patient population with precise evaluations of the roles of IL- 6 in cardiac myxoma patients are warranted.

\section{CONCLUSION}

It has been recognised that IL- 6 predicts tumour growth, tumour recurrence, poor prognosis, or death of patients. IL-6 overproduction is responsible for the inflammatory presentations, constitutional symptoms, and recurrence and distal embolisation of cardiac myxoma. The positive correlation between preoperative IL- 6 values and tumour volumes, and dramatical reduction of circulating IL- 6 values after tumour resection, support the previous hypothesis that cardiac myxoma could be a cellular source of IL-6 release. Surgical resection of cardiac 
myxoma is an absolute choice of eliminating the production of IL-6 in these patients.

\section{CONFLICT OF INTEREST:}

The authors declare that there is no conflict of interest.

\section{AUTHORS' CONTRIBUTION:}

SMY: Contributed to the conception or design of the work; and the acquisition, analysis, and interpretation of data for the work; drafted the work or revised it critically for important intellectual content; finally approved of the version to be published; agreed to be accountable for all aspects of the work in ensuring that questions related to the accuracy and integrity of any part of the work are appropriately investigated and resolved.

HZL: Contributed to the interpretation of data for the work; and finally approved the version to be published.

\section{REFERENCES}

1. Yuan SM, Yan SL, Wu N. Unusual aspects of cardiac myxoma. Anatol J Cardiol 2017; 17(3):241-7.

2. Bushnell JR, Weston C, Karamadoukis L. An unusual presentation of atrial myxoma with haematuria and proteinuria. NDT Plus 2011; 4(2):124-5.

3. Morishima A, Marui A, Shimamoto T, Saji Y, Nishina T, Komeda M. A case of interleukin-6-producing cardiac myxoma resembling multicentric Castleman's disease. J Thorac Cardiovasc Surg 2009; 138(2):499-501.

4. Mendoza CE, Rosado MF, Bernal L. The role of interleukin-6 in cases of cardiac myxoma. Clinical features, immunologic abnormalities, and a possible role in recurrence. Tex Heart Inst J 2001; 28(1):3-7.

5. Koo YH, Kim TG, Kim OJ, Oh SH. Multiple fusiform cerebral aneurysms and highly elevated serum interleukin- 6 in cardiac myxoma. J Korean Neurosurg Soc 2009; 45(6): 394-6.

6. Ezerioha N, Feng W. Intracardiac myxoma, cerebral aneurysms and elevated interleukin-6. Case Rep Neurol 2015; 7(2):152-5.

7. Morishima A, Marui A, Shimamoto T, Saji Y, Nishina T, Komeda M. A case of interleukin-6-producing cardiac myxoma resembling multicentric Castleman's disease. J Thorac Cardiovasc Surg 2009; 138(2):499-501.

8. Nishio $Y$, Ito $Y$, Iguchi $Y$, Sato H. MPO-ANCA-associated pseudovasculitis in cardiac myxoma. Eur J Neurol 2005; 12(8):619-20.

9. Sakamoto $H$, Sakamaki $T$, Sumino $H$, Sawada $Y$, Sato $H$, Sato $M$, et al. Production of endothelin-1 and big endothelin-1 by human cardiac myxoma cells implications of the origin of myxomas. Circ J 2004; 68(12):1230-2.

10. Salobir B, Sabovic M, Kozelj M. Increased levels of antiphospholipid antibodies in a woman with left atrial myxoma and systemic embolisms. Lupus 2001; 10(11): 815-7.

11. Shiraishi I, Yamagishi M, Kato R, Okumura $Y$, Sato $H$, Tanaka $T$, et al. A case in a child of giant left-atrial myxoma associated with recurrent high fever and myxoma cells expressing interleukin 6. EurJ Pediatr 2006; 165(5):346-7.

12. Suzuki J, Takayama K, Mitsui F, Kono T, Yazaki Y, Takei M, et al. Cardiovasc Pathol. In situ interleukin-6 transcription in embryonic nonmuscle myosin heavy chain expressing immature mesenchyme cells of cardiac myxoma. Cardiovasc Pathol 2000; 9(10):33-7.

13. Maze $Y$, Kajimoto M, Tenpaku H, Satou T. Left atrial myxoma with severe inflammatory response. Jpn J Thorac Cardiovasc Surg 2004; 52(4):221-3.

14. Mochizuki $Y$, Okamura $Y$, lida H, Mori H, Shimada K. Interleukin-6 and "complex" cardiac myxoma. Ann Thorac Surg 1998; 66(3):931-3.

15. Yokomuro $\mathrm{H}$, Yoshihara $\mathrm{K}$, Watanabe $\mathrm{Y}$, Shiono N, Koyama $\mathrm{N}$, Takanashi $\mathrm{Y}$. The variations in the immunologic features and interleukin- 6 levels for the surgical treatment of cardiac myxomas. Surg Today 2007; 37(9):750-3.

16. Volz MS, Loddenkemper C, Morguet A, Moos V, Schneider T. Cardiac myxoma secreting interleukin- 6 promotes cavitary tuberculosis: A case report. Int J Tuberc Lung Dis 2015; 19(10):1265-6.

17. Endo A, Ohtahara A, Kinugawa T, Ogino K, Hisatome I, Shigemasa C. Characteristics of cardiac myxoma with constitutional signs: A multicenter study in Japan. Clin Cardiol 2002; 25(8):367-70.

18. Yuan SM, Lin HZ. Predictors of normalization of circulating interleukin- 6 after cardiac myxoma resection. Braz J Cardiovasc Surg 2019; 34(1):22-7.

19. Yuan SM. Cerebral aneurysms due to cardiac myxoma. J Coll Physicians Surg Pak 2019; 29(8):763-7.

20. Yaguchi $H$, Murakami $Y$, Sengoku R, Sato H, Inoue K. A case of cardiac myxoma presenting with multiple cerebellar hemorrhages and elevation of interleukin- 6 in the cerebrospinal fluid. Rinsho Shinkeigaku 2004; 44(10): 677-81.

21. Acebo E, Val-Bernal JF, Gómez-Román JJ, Revuelta JM. Clinicopathologic study and DNA analysis of 37 cardiac myxomas: A 28-year experience. Chest 2003; 123(5): 1379-85.

22. García-Zubiri C, Citores MJ, Yebra-Bango M. Contribution of monocytes to overproduction of interleukin- 6 in a case of cardiac myxoma. Am J Med Sci 2009; 338(4):336-7. 